\title{
Cognition Object and Disagreement Phenomenon
}

\author{
Shulepova Olga Borisovna
}

Associate Professor, Doctor of Philosophy, Professor, Department of Philosophy, Amur State University

E-mail: Shulepova@mail.ru

\section{Doi:10.5901/mjss.2015.v6n2s4p79}

\begin{abstract}
The article deals with social-cultural conditionality of the scientific study object and consideration of the disagreement phenomenon role in its determination based upon natural and humanitarian sciences. The phenomenon of disagreement as the display of mechanism of object determination was traced based upon the facts of history of theoretical extraction and determination of such objects nature as solutions (in chemistry), light (in physics), the Great French Revolution XVIII century and Victorian England (in history).
\end{abstract}

Keywords: scientific research object, classical science, nonclassical and post-nonclassical, social-cultural conditionality of the cognition process, natural and humanitarian sciences, disagreement phenomenon.

\section{Introduction}

From the day of introduction of the notion "object" into philosophy, as such one that is directed on the cognitive activity of cognoscitive (subject), existence of the object was supposed as self-evident, non-problematic and not causing any issues. Science objects are natural, i.e. they exist independently, "on their own", out of position of the cognoscitive subject. Such naïve-naturalistic view about existence of science object corresponded to the ideals of classical science. In this case we rely ourselves on the subdivision of science stages offered by V. S. Stjopin (and, correspondingly, scientific rationality) on the classical, nonclassical and post-nonclassical.

The classical stage of science development dominated up to the beginning of XX century. The views about scientific cognition, peculiar to the classical science, were named by I. Scheffler as "standard conception of science". The content of "standard conception of science" is reduced to the following provisions. The world exists objectively. The aim of science is the study of this objective world. The scientific knowledge is the constant accumulation of true views about this world. Although in the world surrounding humans some changes take place, generally it is uniform in its basis. The confirmation of this uniformity is universal and permanent laws of nature. These laws are learnt by the subject as a result of application of the universal scientific method. The cognition is impassionate observation that provides obtainment of the "clean", objective knowledge, i.e. completely independent on any subjective qualities of the concrete scientists. The development of the scientific knowledge is the cumulative process.

As we can see, the standard conception of science expressed naïve-realistic approach to cognition. Within this approach cognition was understood as direct imitation of the reality, content of the scientific knowledge was deduced from studied reality without taking into account the contribution, done by the culture. The role of culture was limited by the contribution only into form of scientific knowledge.

Naive-realistic consciousness postulates the absolute division of object and subject. From the position of naiverealistic cognitive directions the existence of the science objects was not represented as the special problem. The view about existence of objects in reality "itself" was undoubted. The science object was understood as the fragment of nature "itself", independent on the activity of a human as a researcher. "The researcher-naturalist never asks questions about which "the object" appeared and how it was received, - G. P. Shedrovitskii stated, characterizing the peculiarities of this naïve-naturalistic approach, naturalistic vision way and world view, - as for him, in spite of how methodologically intricate and developed he is, the nature from the very beginning consists of objects, more precisely, as K. Marx wrote, from the objects of contemplation, which become the objects of special scientific study". The direction of classical physics (most developed form of natural science) was statement about absolute character of the research physical phenomenon, i.e. about independence of the study object, its properties on the researcher's effect and experimental means of cognition and counting systems.

In the classical science it was supposed that object appears before the researcher the same one, as it exists in 
nature, not subjected to the effect of the human activity. Exclusively and unambiguously such one as it exists on its own, independently on the human and its activity the nature finally is reflected in the natural scientific knowledge. The standard conception of science allowed possibility to have empirical fixations similar for all scientists - "observation data", obtained by one or other (any) observation, i.e. achievement of general meaning in the individual sensitive perception. From the generalization of empirical material the possibility of direct transition to the theoretical formations was allowed, and such scheme of direction of the scientific research (from observations, experiment and results of their generalizations to notions, principles and ideas, united in the theoretical system), was considered to be the only possible.

The view about cognition objectivity in the classical science was reduced to the claim of the world on its own, and objectivity of knowledge were understood as the correspondence of knowledge independent from the human and its activity to object. The knowledge was considered to be more objective and true, than it was more deprived of individual and personal nature. The ideal, to which the classical science aspired, was cognitive result, maximally released from any social and personal characteristics.

The individual cognitive experience and social-cultural context of cognition was not taken into consideration as both not having principal value. As all the features individualizing subjects are inessential and are not subjected to elimination from the final result, the subject turns out to be one and the same in the cognitive situations. As it was offered that the true result can be achieved by any member of the scientific community, each cognoscitive is comprehensible to the right view about reality. The classical view about truth was connected with one and single incarnation of its existence.

\section{Research Methodology and Findings}

The ideals of classical science, formed initially in the natural science, were imitated in the area of social-humanitarian sciences, oriented on the natural sciences, evaluated as more developed. Although the specific peculiarities of humanitarian sciences gave the objective bases for perception of irremovability of human contribution in the recognition of the studied reality earlier, than in the natural sciences. However, this possibility was not realized due to the general estimation of the natural sciences as such ones, which developed more successfully.

The achievements of science of XX century led to transition of the science in nonclassical (first half of XX century), and then in post-nonclassical century (from the end of XX century up to presently) stage of development. On these stages of science development, social-cultural conditionality of the cognition process became comprehensible, irremovability of human from cognition.

For nonclassical science the interrelationship of the object, means and operation of the subject activity is typical. The creation of relativist, and then quantum model of nature radically changed the theoretical-cognitive perception and style of physicists' thought.

The opening of phenomenon of micro-world, which representation is impossible without taking the role of instruments means, without participation of subjective qualities of observer showed that the studied object mainly can't be separated from the device operation, and this action must be taken into account. In quantum physics the interaction between quantum objects and measuring devices was included into single quantum phenomenon at the description of quantum effects the accounting of conditions is required, at which the data of experiment are obtained.

The relativistic physics comes out from cognition of dependence of the physical objects description from the choice of source counting system, as the range of important phenomenon characteristics depends on this choice, result of interrelation of physical objects in the certain observation or experimental situation is represented differently.

Thus, in nonclassical physics it was found out that the content of theory includes in the taken-off type the result of interaction and interrelation of objects and means of study (accounting systems, experimental means). Nonclassical physics faces the difficulty that was concluded in that as M. Born, "it is possible to speak about the state of the objective world upon the condition that this state depends on what the observer does".

The revolutionary transformation in the natural science of XX century led to the necessity of essential specification and concretization of understanding of the source cognitive relations - interaction of the object and subject of cognition. If in the classical science the absolute character was stated, the independence of objects from subjects and condition of cognition, then now the subject and conditions of cognition were included in the very object. The notion of object of "nonclassical" type, i.e. such object that includes not only the fragment of the objective reality, but also the methods of study, is formed.

Post-nonclassical science takes into account the interrelationship knowledge about object not only with means, but with valuable-target structures of activity, offering explication of intrascientific values and their correlation with social aims and values.

The classical science that states absolute opposition of subject and object, turned out to be far from their views 
about cognition from truth. It didn't take in account that a human is practical being and understands world in that extent, in which he is able to transform it and realize himself in it. The world "itself" serves as the true object of cognition and the world "itself" as it rises before a man in its practical activity. The justice of this provision and insolvency of naïve-realistic view and was detected in connection with achievements of science of XX century.

Nonclassical and post-nonclassical science are derived from the social-cultural conditionality of the cognition process on the whole and object of cognition including. The social-cultural conditionality of the cognition process and its objects means that the scientists look on the object through the prism of theoretical constructions. "... The peculiarity of direct physics subject - physical reality: fusion of the nature material and certain ideal construction, within which the nature material obtains its comprehension, "understandability" for physics."

From the position of social-cultural conditionality of the cognition process the objects of science appear not as predestined by the nature to the scientist, not predetermined by his study, but supposed as such one, extracted from the objective reality in the process of practical effect on it. The "Nature" as universal set of objects of possible scientific experience, - M. B. Sapunov writes, - is not some metaphysical reality initially subjected to curiosity and intellectual curiosity; it is extracted as the day of practical relation of a human to the world and that's why is always socially coloured, it historically appears and evolves together with historical appearance and evolution of science." The subject is provided with very rich choice of possibilities of extraction of the material objects according to the different set of signs. The true object of cognition comes forward not as "itself", and the world, "answering" to a human for his "inquiry" on the practical intervention of human into it. V. Heisenberg marked that physics recognize not simply the nature, but its part, which is revealed owning to the way of asking question about the nature.

Considering the scientific cognition, it is necessary to take into account the difference between objective reality and subjective reality of the science.

Subjective reality is its direct subject of work of the scientist. The subjective reality is abstract, ideal moment of sensible-experimental activity of the subject, objectified in its theoretical and experimental language. This is a view about the world, picture of the world on this or that stage of science development. The subject specification of the notion "subject reality" is physical, astronomic, biological, psychological, historic and other realities.

The object of cognition is not some duty, but some present being, as it is. The object of cognition should be understood as such one that is realized through actualization of its different possibilities of existence. The different socialcultural, individual cognitive experience allows actualizing the different possibilities of existence.

The object, as it exists in the objective reality, reality itself, was not determined as an object of scientific cognition. In the scientific world its determination takes place, i.e. description in science language, its empirical and theoretical extraction from the objective reality, reality itself and thus extraction in the subjective reality. The determination of the object is not some single procedure. Even in the frameworks of one science language we are not able to finish determination of the object. However, in science there exist different languages of empiric and theoretical description in the science.

In the scientific cognition object of study is provided to subject not only through experimental-observance conditions of cognition, but through the specific aggregate of theoretical idealizations - abstract and/or ideal objects. The theoretical constructions reproduce one or other possibility of existence. The experience of the physics development of XX century evidences about possibilities of building of several theoretical systems on the basis of one and the same empirical material or different empirical interpretations of one and the same theory. By means of epistemological analysis it seems possible to choose one of the theoretical conceptions as the true one, and reject all the others as false ones: all variants appearing in this case are equal and have the right for existence and are subjected to substantiation and experimental testing.

In the modern science it is still more recognized that the truth about subject is achieved by the way of dialogic interaction of theoretical constructions, which reproduce one or other possibility of existence. The dialogical type of communication is supposed between competing theories, which co-exist in time, and are remote from each other in time, sometimes were substantial.

In this case the peculiar mechanisms of object determination act, which provide the existence of different languages of description. There happen such situations, when the appearance of new languages of description, and application of different languages of description lead to the views contradicting each other. The displays of mechanisms of object determination in these situations are the phenomenon of disagreement.

The development of the views about reality, which the science deals, recognition of its social-cultural nature leads to understanding of the collective and popular character of cognition subject that allows, in its turn, writing in this model of dialogue and communication relation, including the phenomenon of disagreement. Thus, this phenomenon arises in connection with the new vision of reality in science not as the outside, non-existing for cognition, not as the annoying 
obstacle in the development of scientific knowledge, but as the phenomenon, limitedly inherent to the science, by means of which, in particular, the determination of cognition object happens.

The theoretical relation of a human to the world is varied in different social-cultural and individual cognitive contexts, accordingly the subjects of activity are not similar, they are distinguished from each other, and there are many of them. The different vision of reality by the individual cognition subjects leads to the development of different ontological object pictures. Each concrete scientist is the unique device that enters into unique relation with the object. Nonclassics based upon quantum mechanisms, revealed only the role of technical device. The further development of science allows understanding in the form of unique device and scientist person. The development of the new ontological view sometimes takes place through the disagreement of scientist with the previous views about the object.

We meet the disagreement as the display of object detection mechanism both in the natural and socialhumanitarian substances.

In the area of natural sciences it is possible to remember the history of doctrine about solutions in physical chemistry, which made the important page in the history of chemical science. In the development of views about solutions it was going about the nature of one and the same phenomenon, but chemists gave different ontology of the object of their study.

Since the end of 60-s years of XIX century, hydrated or chemical theory dominated in the physical chemistry. Hydrated theory explained the solution of not only mechanical distribution of the molecules of the soluble body between molecules of dissolvent, but the interaction between them that bears chemical character. According to this view, the dissolvent and solute substance forms associative-dissociative system that is in the movable balance.

In 80-s years of XIX century the renowned Swiss chemist Svante Arrhenius (1859-1927) was created the ion theory of solutions. The development of this theory was also conducted by Ostwald, Nernst and other physical chemists. The bases of electric chemistry, founded by the works of Davy, Berzelius, Faraday and other scientists in the first half of XIX century promoted to the development of ionic theory. They studied effect of the electric current on the chemical compounds and electrolyses phenomena. Solutions were subdivided into electrolytes, i.e. carrying electric current, and non-electrolytes, on which the electric current doesn't effect. At that it was supposed that the current decomposed electrolytes into ions, distinguishing from the neutral particles by their electric charge.

Arrhenius offered principally new explanation of the dissolubility, coming in the context with views dominating in chemistry. In accordance with Arrhenius point of view, the electrolyte in solution was partially dissociated into ions; so the current is not the reason of dissolution of the neutral molecules on the oppositely charged ions, and on the contrary, its carrying through the solution of electrolyte is the consequence of ion presence in the solution.

Hydrated theory denied dissociation of molecules into ions and stated that electric conductivity of solutions, as a rule, is reasoned by formation of the corresponding complexes between dissolvent and solute substances. According to the ionic theory the soluble molecules are partially dissociated into ions, which are in balance with the non-dissociated molecules, at that the balance is not shifted in the part of dissociation of the bigger number of molecules at the increase of solution dilution.

If the hydrate theory explained the properties of solutions by the character of interaction of dissolvent with solute substance, then the ionic theory completely or in any case predominantly by the nature of solute substance (electrolyte). The ionic theory adequately described the behaviour of diluted solution, and in this relation has undoubted advantages over hydrated theory, however it was insufficiently effective in the explanation of concentrated solutions.

The number of opponents to the new brave theory, which belonged to the most renowned and authoritative chemist of that time, immediately appeared. They considered the theory of electric dissociation to be false and absurd.

The critical non-acceptance of the theory of electrolytic dissociation was explained by that the chemists relied themselves upon that recognition of reality of ions, identification of notions "atom" and "ion" can result in reconsideration of the point of view on the atom nature as an absolute unchangeable particle, and the idea of atom indivisibility and its invariability in different compounds of them was the fundamental in science. In the theory of electrolytic dissociation Arrhenius' colleagues saw the threat of undermining the basis of science modern for them.

In 90-s years of XX century, as it was marked by the historian of science Yu. I. Solov'ev on the chemical conventions and conferences, the polemic cyclone literally rushed over pages of scientific journals. The further development of the theory of solutions (electrolytes) proceeded in the direction of overcoming of one-sided ionic and hydrate conceptions, in the direction of their theoretical synthesis.

The phenomenon of disagreement served as a display of object detection mechanism and at the theoretical extraction and determination of the physical nature of such complex and difficult object for study as the light is.

Although the light was studied still from the ancient times, but the development of first theories of light is related to the new time. Approximately in the mid of XVII century, two theories about light nature as corpuscular and wave 
appeared.

According to the corpuscular theory, the light represents the flow of particles emitted by the luminous bodies in all directions - corpuscles, some similarity of "light atoms". The wave theory considered the light as the distribution of the waves in ether.

Corpuscular views on the light nature into the harmonious theory were developed by Isaac Newton (1643-1727). Newton supposed that the movement of light corpuscles, which were moved in the space with huge speed, until they meet obstacle can be explained by the mechanics law. Corpuscular theory explained well the law of direct light distribution, its reflection and refraction. Linearity is the display of the first law of dynamics: light corpuscles move evenly and directly, if they are not affected by any forces. Newton understood the reflection of the light according to analogy with bouncing the resilient ball from the plane, refraction of light as the change of corpuscles speed at transition from one environment into the other one.

Newton also showed that the white light is the composite and contains "pure colours", each characterizing its refraction. This peculiarity was explained also from the positions of corpuscular interpretation of the light nature. Corpuscles have different weight and size, and it conditions their colouring. Getting on the eye retina, the small-sized corpuscles create impression of dark-red colouring, and the smallest ones - of violet colour. Corpuscles, varied in their size, experience different refractions at transit from one optic dense environment into the other one. According to Newton's idea it followed that the speed of light in the substance is more than the speed of light in the vacuum.

Owing to the huge Newton's scientific authority, the corpuscular theory became the dominating one, and the whole XVIII age passed in physics under the sign of this theory. Indeed, the modern scientists, and first of all, S. I. Vavilov, faithfully object to the single-meaning Newton's interpretation as unconditional supporter of corpuscular theory. S. I. Vavilov marked that: "...It hardly that among Newton's contemporaries included Huygens and Hooke, were physicists, who so distinctly understood all the benefit of wave theory and its basic features". [9, 70] Vavilov refers to the compromise offer of Newton, in which the advantages of wave and corpuscular theory are merged, writes about width and ingenious sagacity of Newton, who intuitively guessed the two-sidedness of light phenomena in XVII century. Even if to agree with such characteristics of Newton's views, we can't help of recognizing that the dominance of corpuscular theory developed in the science inhibited the development of wave theory.

However, already then, when Newton in 1672 year represented memoir in the Royal Society "A New Theory of Light and Colours", in which he stated the results of his optic studied, based upon experiences, from the positions of corpuscular approach: the conclusion about complexity of white light, presence of simple colours, differentiation of physiological and physical colourity, inseparable connection of the refraction degree with light, and this work caused big disputes. The conclusions, represented by Newton, were completely new and unexpected for his contemporaries, and the Royal Society chose commission of three persons: astronomer Sat Word, physicists Robert Boyle and Robert Hooke for consideration of the manuscript submitted.

In several days Robert Hooke (1635-1703) represented his properly motivated critical review about Newton's work. Further Hooke came forward as permanent critic and opponent to Newton. Marking the thoroughness and refinement of Newton's experiences, which led him to the asserted conclusions, Hooke wrote that these experiences are explained by the wave theory too. He disagree that the colour is inseparable and initial property of rays. According to Hooke's words to affirm that all colours are contained as such ones in the simple light rays would be the same as to speak about presence of all sound tones in the air, concluded in the organ furs or in string, from which they are produced by pressure or hits. Decomposition of the white colour in prism is caused, according to Hooke, by indignation of the simple wave movement in prism.

The critics of Newton's theory almost simultaneously with Hooke were Huygens, Jesuits Pardis, Linus and etc.

The developed disputes took the very desperate character that Newton - a human of very irritable and sensible to critics refused from its continuation. In the letter from June 23 in 1673 Newton wrote to Oldenburg - a secretary of the Royal Society that he didn't want to be engaged in the natural sciences and refused to answer on the critical articles and letters, and that he didn't published works on optics up to Hooke's death.

Criticizing the disadvantages of corpuscular theory, such contemporaries of Newton, as Hooke and Huygens, developed the wave theory. The wave theory in XVIII had thousands of opponents and the little number of supporters. The guess about wave number of the light was told earlier by the Italian physics and astronomer Francesco Grimaldi (1618-1663). He compared the light distribution with distribution of waves on the water.

The idea of wave nature of light was supported by Hooke. In his work, devoted to optics, - "Micrograph" (1665) he wrote that the light represents the quick and vibration movements of some transparent environment, through which it comes. Coming forward with hypothesis about light nature in the Royal Society seven years after, he stated that the light in ether is the same as the light in air. 
The hypothesis stated by Hooke about wave nature of light received the development in the works of Huygens, Fresnel and Young. Hooke as the researcher with very wide interests often put many the most important problems that prevent him to concentrate on one, stated the ingenious idea, made discoveries, but didn't lead many works to the end. Not Hooke, but Christian Huygens (1629-1695) - Netherlands physicist, mechanical engineer and astronomer developed the wave theory of light. At him the wave nature of the light is already completely scientifically substantiated theory. Huygens stated the theory of lights in 1678 in "Treatise about Light" (published in 1690).

The work of Huygens begins from critics of the previous theories of Deckart and Newton. Huygens states the following arguments against corpuscular understanding of the light nature. If the light consists of corpuscles then how can it be distributed in the straight line in the bodies without feeling deviations? How can it be that two cross-cut beams of light, i.e. two flows of particles didn't incite each other by the way of mutual co-hits? It is enough to remember, - as Huygens writes, - that the light appears from the fire and flame, i.e. from the bodies, which are in very quick movement; that the light, concentrated by the mirror, is able to burn subjects, i.e. divide their parts, and this serves a convincing sign of movement; that the visual sensation appears at indignation of the ending of optic nerve; that, as in the case of co-hits, two or several movements can be imposed on without indignation of each other; that the distribution of sound happens by means of movement.

Properly in mathematical way, Huygens showed how the direct distribution of the light, its reflection and refraction take place. In the stated treatise Ch. Huygens formed the principle, according to which each point of space, which distributing wave achieved in this moment becomes the source of elementary spherical waves, and on its bases he produced the laws of light reflection and refraction. Huygens established the phenomenon of light polarization phenomenon that happens to the light beam at its reflection and refraction (especially at the double refraction) and lies in that the fluctuation movement in all points takes place in one plane, passing thought the beam direction, while in the nonpolarized beam the fluctuations take place in all directions perpendicularly to the light. The principle of Huygens allows understanding, why and how deviations take place from the prescriptions of the geometric optics. Having developed the idea of Grimaldi about that the light is distributed not only in the straight line with refraction and reflection, but with fragmentation (diffraction), Huygens gave explanation to all known optic phenomenon. He states that the light waves are distributed in ether that represents the subtle matter, penetrating all bodies.

Thus, to the beginning of XVIII century two opposite approaches to explanation of the light nature existed: corpuscular theory of Newton and wave theory of Huygens. All XVIII century was the age of supporters' fight for these two theories. Both one and the other theories explained basic optic conditionalities: linearity of distribution, laws of reflection and refraction. However, one and the other theory faced with difficulties at explanation of the optical phenomena range.

The explanation corpuscular theory of reflection faced with the difficulty of answering the issue, why only the part of light is reflected, and the other is absorbed by the reflecting body, that happens here. The view about reflected particles led to recognition of that the reflected particles must inevitably hit with the falling ones. Arthur Schopenhauer mocked in this connection over corpuscular theory, stating that it doesn't give possibility to see own physiognomy in the mirror.

The disadvantages of the corpuscular theory of Newton were that it couldn't explain the interference and dispersion of light, and also answer the issue, why at falling on the boundary of two mediums the partial reflection and refraction take place. One of the arguments against corpuscular theory was that all attempts to measure the pressure of light, which naturally the illuminated bodies must experience from the flow of rushing particles, remained ineffective.

The wave theory of Huygens couldn't explain the physical reasons of presence of different colours and mechanism of light distribution speed change in the ether, penetrating into different mediums.

One more disadvantage of the wave theory was connected with that this theory allowed existence of ether - the medium, in which the light is distributed. Resistance on the part of ether must noticeable inhibit the movement of heavenly bodies, but the movement of planets and comets in the sky space doesn't face evident resistance. This circumstance allowed putting the existence of ether through which the light distribution was supposed, and also the truth of wave theory into question. It was impossible to suppose the fact that waves can have not mechanic nature in XVIII century.

However, on the turn of XVIII and XIX centuries the wave theory triumphed. The achievements of wave theory in that time were the merit of English physicist Thomas Young (1773-1829) and French physicist Augustine-Jean Fresnel (1788-1827).

Young added Huygens's principles with the idea of interference, i.e. enhancement or weakening of ways at their imposition. He explained the physical nature of this phenomenon; the term "interference" also belongs to him. Interference is one of the brightest displays of wave properties; it has the important place in the clarification of physical nature of the light. Young distributed the wave views beyond the limits of optics: he guessed that the light and radiant 
heat has the similar physical nature and distributed view about interference on the ultraviolet region. In 1803 Young specified the new approach to the quantitative description of diffraction. Young was the first one, who offered the statement about transverse character of light waves, however frankly speaking, he said about it as of imaginable transverse movement, i.e. as about notion of purely fantastical, so senselessly the transverse fluctuation of ethers were understood by the scientists from the mechanical point of view.

In 1621 Fresnel proved the transverse character of light waves, studied the interference of polarized light, and in 1823 he opened elliptic and circular polarization, having explained the chromatic polarization and rotation of polarization plane, and double beam refraction. Yet before the appearance of electromagnetic theory of Maxwell, Fresnel wrote the famous formulas, which quantitatively described reflection and refraction of the light on the limit of mediums division.

While the wave theory successfully explained optic phenomena, the corpuscular theory was imaginably complicated. Its supporters were forced to introduce completely unsubstantiated and sometimes contradictory hypotheses one by one for interpretation of the phenomena discovered at the beginning of XIX century.

In connection with the works of Young and Fresnel the victory temporarily passed to the wave theory. The achievement of the victory of wave optic in XIX century was promoted by detection of facts, pointing on the connection and analogy of optic and electric phenomena. Faraday, Maxwell and other scientists showed that the light is the private case of electromagnetic wave.

However, in spite of the huge successes in electromagnetic theory of light, the new facts, contradicting the theory of light were accumulated to the end of XIX century. The wave theory could explain the distribution of energy in the specter of emission of absolutely black body and phenomenon of photoeffect.

In 1900 Max Planck (1858-1947) showed that the emission of absolutely black body can be explained, if to suppose that the light is emitted not uninterruptedly, but in portions and quanta.

In 1905 Albert Einstein (1879-1955) explained the conditionalities of photoeffect on the basis of view about light particles - "quanta" of light and "photons".

The works of Planck and Einstein serve the beginning of quantum physics development. In the modern physical optics quantum views don't contradict to the wave ones, but are combined on the basis of quantum mechanics and quantum electrodynamics. The scientists of our time took place from the view about that the light is both waves and corpuscles at the same time.

Thus, in the history of physics both theories - wave and quantum one - developed simultaneously, having its undoubted advantages and disadvantages, and seemingly add each other. Thus because of disagreement of some scientists with the theory of physics generally recognized on one or other stage of development, such object as light was extracted.

The phenomenon of disagreement played huge role in formation of views about historical reality in the historical science.

In the historical study the scientist deals with the past, and historical reality that can't be perceived directly, as in fact it doesn't no already exist. The historian doesn't have possibility to correlate his knowledge with the studied object neither by means of contemplation, not by means of experimental operation practical to it the past is not reproducible. The methodology of cognitive theoretical reconstruction dominates here. A lot of depends on the personality of a historian, his ability to reproduce, and describe "the deeds of long time ago days" at the marked peculiarities of the object of historical cognition.

Based upon example of the historical science the difference of objective and subjective reality is well seen. The scientist-historian evidently deals not with objective reality, not with the past itself, but with the certain way empirically and theoretically described reality.

Certainly, the historical cognition, as well as any science cognition, has constructive character. However, this construction is not voluntary. It is determined by the "address" of historian, to which the cognitive result correlates. We mean its social-cultural and individual context of work, determining one or other cognitive result, under the "address" of historian.

The historian in his study imposes imprint on the studied reality as he always perceives past from the positions of its time, through the prism of modern of social-cultural environment contemporary to it, that inevitable effect on its research position and understanding of the events of the historian past. Moreover, each historian - is not simple the product of its time, he is the unique crossing of social-cultural traditions, along with the social one the historian has the individual context of its work. The individual peculiarities of the research activity are also caused on its results.

The variety of possible approaches, opinions and interpretation of the historical process, existing in the historian science, impose imprint on formation of the representation about subjective reality to which the historian deals.

It is possible to trace the role of disagreement as the display of mechanism of determination of the object in its 
historical study on the example of historiography of XX century about the Great French Revolution of XVIII century. The foreign and native researchers of historiography of the French Revolution mark the polemic character of this historiography and its transformation in the highlighting revolution from "down" and "left" (from social-economic problems) to the problems of mass cognition, to the history of frame of mind, or as F. Dossa writes from "cellar" to "attic".

Initially, till the end of 50-s years of XX century, so-called "classical" approach dominated in the historiography of the French revolution. The development of "classical" conception of the French Revolution in XX century was made first of all by J. Lefevre and E. D. Labrousse, and also disciples of J. Lefevre, A. Soboul, Suratteau, R. Cobb, D. Rude. The international Marxist historiography also developed within the framework of "classical" interpretation of revolution. The "classical" understanding of the French Revolution formed on the basic of methodological approach, which essence was expressed by J. Lefevre in the words: "It is not enough to describe, it is necessary to count". On the basis of explanation the quantitative data received from the serial sources were received. The quantitative data served the important proof in the historical explanation.

The basic attention of the historians in the study of French Revolution was directed on its social-economic part. They were interested in the social-economic structures of the society and social classes. The revolution was interpreted as the highest display of classical fight. At that the key role of revolution was given to the bourgeoisie, even if other layers (aristocracy, peasantry, "small people") played its role in the revolutionary fight. Due to this the traditional understanding of the essence of this revolution as anti-feudal, that lead to unchangeable word usage "The Great French Bourgeoisie revolution". The bourgeoisie character of this revolution made the very core of understanding of its nature, i.e. entered into the content of notion "The Great French Bourgeoisie Revolution".

However, at the end of 50-s years in the historiography the new direction began to form, received the name "critical" or "revisionist". The representatives of this direction (A. Cobben, E. Le Roi Ladurie, D. Riche, J. Taylor, F. Furet and other) stand out with the decisive critics of "classical" conception of revolution. F. Furet calls the theoretical constructions of historians-Marxists "metaphysic monster" that suffocates historical reality with its feelers that is displayed in the pulsatory and unpredictable spontaneity of the revolutionary crisis.

The French historians of "revisionist" direction of F. Furet and D. Riche rejected the traditional "classical", from their point of view, old view of revolution of the end of XVIII century as one of the "single revolution", single social burst, stood out against interpretation of the revolution as bourgeoisie that predetermined inevitability of transit from the feudalism to capitalism. They offered new interpretation of the French Revolution. F. Furet and D. Riche single out in the French Revolution three completely different revolution as revolution of the liberal gentry and bourgeoisie, which answer the interests of capitalistic development: peasant revolution, not so anti-feudal, as anti-bourgeois; sans-culottes revolution, hostile to capitalism and that's why reactionary in its essence. Furet and Riche considered French Revolution as such one, which had fatal consequences for the development of capitalism.

The appearance of works of the representative of "critical" direction caused animated discussion, alarmed the thoughts of historians and appealed their attention to the most important issues about the character of French Revolution, its moving forces and meaning. In the epistemological sense that interests us we can state that the representatives of the "critical" direction in historiography, having paid attention on the well-known phenomenon - French Revolution conducted the new construction of this object. They describe the known phenomenology with the new theoretical language, built the new theoretical model of this object, excluding the possibility of compulsory joining of the term "bourgeoisie" to the words of "French revolution". Now the content of notion of "The Great French Revolution" becomes at least trinomial construction.

In the considered example the disagreement is expressed by the representatives of the whole scientific direction. The historiography contains the examples and individual disagreement, opposition to the general opinion, which promote to the new determination of the object.

E. Briggs is one of the most famous of the representatives of "new historical science" in the Great Britain, the largest authority in the history of Victorian England in connection to the methodology of their work wrote that having convinced that perception of their position by the people often doesn't coincide with the objective data, he passed to the social history as the economic history, connected with the political, social and cultural history. The advantages of this new approach in that time for British historiography he realized in the works of "Victorians", the re-evaluation of characters, institutes, ideas and events" (1954) and "The Age of improvement 1783-1867" (1959). Briggs characterized the object of their researches - Victorian England - as terra incognita, in spite on the reliable tradition of its study. Some historians stood out with critics of "Victorian Values", as false and hypocritical, others, oppositely, saw in the Mid Victorian period the lost moral values and spiritual asylum.

In his researches Briggs refused from the generally accepted views and came to conclusion that the single Victorian England didn't exist yet. As Briggs marks in spite on the brightly expressed cultural succession, there was a little 
unity in the Victorian England. He tried to detect the single system of values, but detected a number of subcultures, frequently contrasting. Briggs states the neither statistical, nor verbal evidences, nor illustrative material give bases for interpretation of the Victorian culture in the unique key.

Thus, having refused from the traditional approaches and views, Briggs could see Victorian England not as homogenous formation, but as the aggregate of subcultures that lead to the change of content of notion "Victorian England", and consequently to the new object of historical study in the cognitive activity of Briggs.

\section{Concluding Remarks}

The appeal to historiography allows after Dorn making a conclusion that: "There is no single way of writing the history or single way of interpretation of the historian sources, as there is no single way to read history.

As in other sciences, the history has not solid ground, strict and eternal truths. We never know "how it was in reality", but we could come closer to the ideal by means of endless dispute between different theories about historical sources. The logics give us the rules of game not for proving anything, but as a system with the help of which it is possible to reject unsubstantiated hypothesis. The theory of discussion offers the set of formal procedures in order to conduct competition of the scientific statements."

Thus, as in the natural-scientific, both in the social-humanitarian cognition the phenomenon of disagreement serves as the display of object detection mechanism of the scientific study. Thus, the similarity evidences for the favour of unity of these two areas of cognition, for favour of problem solution of the humanitarian science status as methodologically isomorphic natural sciences.

\section{References}

Axiological aspects of science development. (1990). Moscow: Nauka.

Blumenau, S. G. (1995). From social-economic historian to the problems of mass consciousness: French historiography of revolution of the end of XVIII century (1945-1993). Briansk: Publ.-house of Briansk State Ped. Un-ty.

Born M. (1963). Philosophical aspects of modern (pp. 81). Moscow.

Briggs, A. (1985-1986). Collected Essays. Vol. II, XV. Urbana.

Classics, nonclassics, post-nonclassics. (2009). Post-nonclassics: philosophy, science, culture. Saint Petersburg: Mir.

Disputes about revolution in the French historical science of second half of 60-70-ss years. (1994). Briansk: Publ.-house of Briansk State Ped. Un-ty.

Dorn, P. (1997). Again about methodology. Old and beautiful: "soap opera" and about non-understanding between historians and models. New and newest history, 3, 94 .

Dosse, F. (1987). L'histoire en miettes: Des "Annales" a la "nouvelle histoire". Paris.

Furet F. (1998). Understanding of French Revolution. Saint Petersburg: INAPRESS.

Heisenberg, V. (1963). Physics and philosophy. Moscow: Publ.-house of foreign literature.

Huygens, Ch. (1973). Treatise about light (pp. 21-35). Ch. Huygens. Creators of physical optics. Moscow: Nauka.

Kosareva, L.M. (1980). Object of science: cognition and comprehension (Introductory article). Moscow: INION AS USSR.

"Revisionist" direction in the modern French historiography of the Great bourgeoisie revolution of the end of XVIII century. (1992). Briansk: Publ.-house of Briansk State Ped. Un-ty.

Sapunov, B. M. (2012). Problem of reality in history and philosophy of science. Higher education in Russia, 2,148.

Shchedrovitskii G. P. (1995). Methodological sense of opposition of naturalistic and system-active approaches. Shchedrovitskii G. P. Selected works. Moscow.

Solov'ev. Yu. (1990). I. Svante Arrenius. 1859-1927. Moscow: Nauka.

Stepin V. S. (1989). Scientific knowledge and values of technogenic civilization. Issues of philosophy, 10, 3-8.

Vavilov, S. I. (1989). Isaac Newton. 1643-1727. Moscow: Nauka. 
